\title{
EFFECT OF THE TEMPORAL PROFILE OF THE FRICTION POWER ON TEMPERATURE OF A PAD-DISC BRAKE SYSTEM
}

\author{
Aleksander Yevtushenko, Michą Kuciej, Katarzyna Topczewska \\ Bialystok University of Technology, Faculty of Mechanical Engineering, Bialystok, Poland \\ e-mail:m.kuciej@pb.edu.pl
}

\begin{abstract}
A mathematical model to analyse the influence of change of the friction power over time on temperature of a pad-disc tribosystem has been proposed. For this purpose, a boundary-value problem of heat conduction for two semi-infinite bodies with taking into account heat generation due to friction on the contact surface has been formulated. Exact solutions of this problem were obtained for seven temporal profiles of the specific heat generation power, which were established experimentally. For selected friction materials, numerical analysis of the spatiotemporal temperature distributions and heat fluxes intensities were executed. The obtained results were compared with a corresponding data which were found by means of the known approximate solution.
\end{abstract}

Keywords: braking, friction power, frictional heating, temperature, heat flux

\section{Introduction}

One-dimensional mathematical models of frictional heating processes in tribological systems are formulated on the basis of domination of heat spreading along the normal to a contact surface into elements of a friction pair (Fazekas, 1953; Blok, 1955; Newcomb and Spurr, 1967). In pad-disc braking systems, three schemes of contact are commonly selected: semi-space-semi-space, strip-semi-space, and strip-strip. On the friction surface of the mentioned elements, one of two variants of the boundary condition of thermal friction contact are assumed: perfect and imperfect one. For both variants, a condition is common that the sum of intensities of heat fluxes directed to the inside of the friction elements along the normal to the contact surface is equal to the specific power of friction $q(t)=f p(t) V(t)$, where $f$ is the coefficient of friction, $p$ is contact pressure, $V$ is sliding speed, $t$ is time (Ling, 1959). The second boundary condition assumes the equality of friction surfaces temperature (perfect contact) or a temperature jump due to interfacial thermal resistance (imperfect contact) (Barber and Comninou, 1989). More detailed information regarding the statement of thermal problems of friction are contained in the article by Yevtushenko et al. (2013).

A review of achievements relating to the methods of formulating and solving thermal problems of friction during braking with the above-mentioned schemes and boundary conditions, i.e. one-dimensional boundary-value problems of heat conduction with account of frictional heat generation, was presented in the article by Yevtushenko and Kuciej (2012). In this paper, the semi-space-semi-space scheme is considered, therefore, corresponding achievements are presented exclusively for this tribosystem. The analytical solution to the thermal problem of friction for two semi-spaces with a constant specific friction power and equality of temperature on the contact surfaces of sliding bodies was presented in the monograph by Carslaw and Jaeger (1959), and a similar problem with account of contact heat exchange and $q=$ const in Sazonov (2006, 2008). Frictional heating during sliding of two homogeneous semi-spaces with constant deceleration and pressure under the condition of imperfect thermal friction contact was examined in 
Nosko and Nosko (2006), and exponential growth of contact pressure from zero at the initial moment to the nominal value at the stopping moment, in the study by Yevtushenko et al. (1999). Generalization of this problem to the case when one semi-infinite body is a composite laminate was investigated in the paper by Matysiak et al. (2002), and the influence of thermosensitivity of semi-space materials on temperature of a braking system in Yevtushenko et al. (2014a,b, 2016).

Basing on the mentioned above investigations, it has been established that friction and wear characteristics of frictional elements of braking systems can considerably differ depending on the manner of absorbing braking energy by such systems. There are various cases possible: in some of them the friction work is distributed more uniformly with time, and in others the main part of the braking energy is accomplished during the initial period of the process. The temporal profile of the friction work during braking is determined by the time profile of the friction power. This profile is the primary factor responsible for spatiotemporal distributions of temperature in friction elements, its maximum values and gradients in the direction perpendicular to the surface of friction.

The solution to the boundary-value problem of heat conduction for a semi-space heated on the surface by a heat flux with an intensity proportional to different, empirically established, temporal profiles of the specific friction power was obtained by Yevtushenko et al. (2017). In the present study, the corresponding exact solutions to the heat conduction problem for two semi-spaces with account of frictional heat generation on the contact surface will be obtained. A significant difference of this paper from the above mentioned is that the intensities of the heat fluxes are not given a priori, but are determined from the solution to the problem.

\section{Statement to the problem}

The classification of ten functions $q_{i}(t), i=1,2, \ldots, 10$, which describe the change in the specific power of friction during single braking from the initial time moment $t=0$ to the stopping moment $t=t_{s}$ was presented in the monograph by Chichinadze (1967). The processes of braking assumed that the total work of friction $w_{0}$ for each function $q_{i}(t)$ was the same

$$
\int_{0}^{t_{s}} q_{i}(t) d t=w_{0} \quad i=1,2, \ldots, 10
$$

Taking into account formula (2.1), we write the functions $q_{i}(t)$ in the following form (Yevtushenko et al., 2017)

$$
q_{i}(t)=q_{0} q_{i}^{*}(t) \quad q_{0}=\frac{w_{0}}{t_{s}} \quad 0 \leqslant t \leqslant t_{s} \quad i=1,2, \ldots, 10
$$

where

$$
\begin{array}{lrrr}
q_{1}^{*}(t) & =2\left(1-t^{*}\right) & q_{2}^{*}(t)=2 t^{*} & \\
q_{3}(t) & =1.5 \sqrt{1-t^{*}} \cong 1.5\left(1-0.5 t^{*}-0.125 t^{* 2}-0.0625 t^{* 3}\right) & q_{4}^{*}(t)=1.5 \sqrt{t^{*}} \\
q_{5}^{*}(t) & =3 t^{* 2} & q_{6}^{*}(t)=1.2\left(1-t^{*}\right)\left(1+2 t^{*}\right) & q_{7}^{*}(t)=1.2 t^{*}\left(3-2 t^{*}\right) \\
q_{8}^{*}(t)=3\left(1-t^{*}\right)^{2} & q_{9}^{*}(t)=6 t^{*}\left(1-t^{*}\right) & q_{10}^{*}(t)=6 \sqrt{t^{*}}\left(1-\sqrt{t^{*}}\right)
\end{array}
$$

and $t^{*}=t / t_{s}$.

The distribution of transient temperature fields $T_{l, i}(z, t)$ in the disc $(l=1)$ and in the pad $(l=2)$ corresponding to the specific power of friction $q_{i}(t), i=1,2, \ldots, 10(2.2)$ and $(2.3)$ 
are found from the solution of the following one-dimensional boundary-value problem of heat conduction for two semi-spaces in perfect friction contact $\left(0<t \leqslant t_{s}\right)$

$$
\begin{aligned}
& \frac{\partial^{2} T_{1, i}(z, t)}{\partial z^{2}}=\frac{1}{k_{1}} \frac{\partial T_{1, i}(z, t)}{\partial t} \quad z>0 \quad \frac{\partial^{2} T_{2, i}(z, t)}{\partial z^{2}}=\frac{1}{k_{2}} \frac{\partial T_{2, i}(z, t)}{\partial t} \quad z<0 \\
& \left.K_{2} \frac{\partial T_{2, i}(z, t)}{\partial z}\right|_{z=0}-\left.K_{1} \frac{\partial T_{1, i}(z, t)}{\partial z}\right|_{z=0}=q_{i}(t) \\
& T_{1, i}(0, t)=T_{2, i}(0, t) \equiv T_{i}(t) \quad \quad|z| \rightarrow \infty \quad l=1,2 \\
& T_{l, i}(z, t) \rightarrow T_{a} \quad \mid=\infty
\end{aligned}
$$

and

$$
T_{l, i}(z, 0)=T_{a} \quad|z|<\infty \quad l=1,2
$$

where $z$ is the spatial coordinate, $T_{i}$ - temperature of the contact surface, $T_{a}$ - ambient temperature, $K_{l}$ - thermal conductivity, $k_{l}$ - thermal diffusivity of the disc and the pad materials.

Denoting

$$
\begin{array}{lccc}
\zeta=\frac{z}{a} & \tau=\frac{k_{1} t}{a^{2}} \quad \tau_{s}=\frac{k_{1} t_{s}}{a^{2}} \quad K^{*}=\frac{K_{2}}{K_{1}} & k^{*}=\frac{k_{2}}{k_{1}} \\
T_{0}=\frac{q_{0} a}{K_{1}} \quad T_{l, i}^{*}=\frac{T_{l, i}-T_{a}}{T_{0}} \quad T_{i}^{*}=\frac{T_{i}-T_{a}}{T_{0}} & l=1,2 \\
i=1,2, \ldots, 10
\end{array}
$$

$a=\max \left\{a_{1}, a_{2}\right\}, a_{l}=\sqrt{3 k_{l} t_{s}}, l=1,2$ - effective depth of heat penetration (Chichinadze et al., 1979), we write boundary-value heat conduction problems (2.4) and (2.5) in the dimensionless form $\left(0<\tau \leqslant \tau_{s}\right)$

$$
\begin{aligned}
& \frac{\partial^{2} T_{1, i}^{*}(\zeta, \tau)}{\partial \zeta^{2}}=\frac{\partial T_{1, i}^{*}(\zeta, \tau)}{\partial \tau} \quad \zeta>0 \quad \frac{\partial^{2} T_{2, i}^{*}(\zeta, \tau)}{\partial \zeta^{2}}=\frac{1}{k^{*}} \frac{\partial T_{2, i}^{*}(\zeta, \tau)}{\partial \tau} \quad \zeta<0 \\
& \left.K \frac{\partial T_{2, i}^{*}(\zeta, \tau)}{\partial \zeta}\right|_{\zeta=0}-\left.\frac{\partial T_{1, i}^{*}(\zeta, \tau)}{\partial \zeta}\right|_{\zeta=0}=q_{i}^{*}(\tau) \\
& T_{1, i}^{*}(0, \tau)=T_{2, i}^{*}(0, \tau) \equiv T_{i}^{*}(\tau) \quad T_{l, i}^{*}(\zeta, \tau) \rightarrow 0 \quad|\zeta| \rightarrow \infty \quad l=1,2
\end{aligned}
$$

and

$$
T_{l, i}(\zeta, 0)=0 \quad|\zeta|<\infty \quad l=1,2
$$

where the dimensionless temporal profiles of the specific friction power $q_{i}^{*}(\tau)$ were determined from formulas $(2.3)$ by replacing in the functions $q_{i}(t)$ of time $t$, braking time $t_{s}$ and ratio $t^{*}$ with the corresponding dimensionless parameters $\tau, \tau_{s}$ and $\tau^{*}=\tau / \tau_{s}$, respectively. It should be noted that solutions to problems (2.4) and (2.5) for the three profiles $q_{i}(t), i=8,9,10$ (2.3) were obtained by Topczewska (2017). Therefore, in this study, we consider functions $q_{i}(t)$, $i=1,2, \ldots, 7$, Eqs. (2.3), only.

\section{Exact solution to the problem}

In the case with a constant specific power of friction $q_{0}^{*}(\tau)=1,0 \leqslant \tau \leqslant \tau_{s}$, the solution to boundary-value problem of heat condition (2.7) and (2.8) is known (Carslaw and Jaeger, 1959)

$$
T_{l, 0}(\zeta, \tau)=2 \gamma \sqrt{\tau} \operatorname{ierfc} Z_{l}(\zeta, \tau) \quad 0 \leqslant \tau \leqslant \tau_{s} \quad l=1,2
$$


where

$$
\begin{aligned}
& Z_{1}(\zeta, \tau)=\frac{\zeta}{2 \sqrt{\tau}} \quad \zeta \geqslant 0 \quad Z_{2}(\zeta, \tau)=-\frac{\zeta}{2 \sqrt{k^{*} \tau}} \quad \zeta \leqslant 0 \\
& \gamma=\frac{1}{1+\varepsilon} \quad \varepsilon=\frac{K^{*}}{\sqrt{k^{*}}}
\end{aligned}
$$

$\operatorname{ierfc}(x)=\sqrt{\pi} \exp \left(-x^{2}\right)-x \operatorname{erfc}(x), \operatorname{erfc}(x)=1-\operatorname{erf}(x), \operatorname{erf}(x)-$ Gauss error function. Then, using Duhamel's theorem, the solution to problems (2.7) and (2.8) for functions $q_{i}^{*}(\tau)$, $i=1,2, \ldots, 7$ are presented in the form (Luikov, 1968)

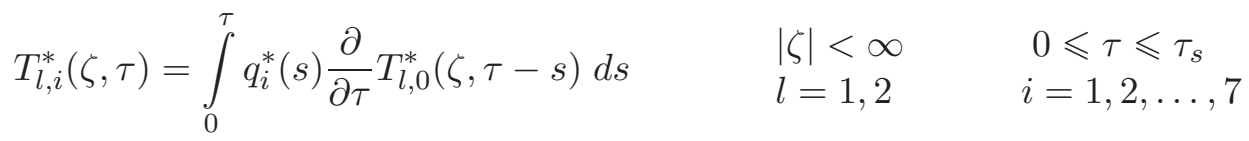

Taking under consideration the forms of functions $q_{i}^{*}(\tau)(2.2)$ and (2.3) and the partial derivative (Abramowitz and Stegun, 1964)

$$
\frac{\partial}{\partial \tau} T_{l, 0}^{*}(\zeta, \tau-s)=\gamma \frac{\exp \left[-Z_{l}^{2}(\zeta, \tau-s)\right]}{\sqrt{\pi(\tau-s)}}
$$

in solution (3.3), the dimensionless temperatures $T_{l, i}^{*}(\zeta, \tau),|\zeta|<\infty, 0 \leqslant \tau \leqslant \tau_{s}, l=1,2$, $i=1,2, \ldots, 7$, are written as

$$
\begin{aligned}
& T_{l, 1}^{*}(\zeta, \tau)=2 \gamma\left[I_{l}^{(0)}(\zeta, \tau)-I_{l}^{(1)}(\zeta, \tau)\right] \quad T_{l, 2}^{*}(\zeta, \tau)=2 \gamma I_{l}^{(1)}(\zeta, \tau) \\
& T_{l, 3}^{*}(\zeta, \tau)=1.5 \gamma\left[I_{l}^{(0)}(\zeta, \tau)-0.5 I_{l}^{(1)}(\zeta, \tau)-0.125 I_{l}^{(2)}(\zeta, \tau)-0.0625 I_{l}^{(3)}(\zeta, \tau)\right] \\
& T_{l, 4}^{*}(\zeta, \tau)=1.5 \gamma I_{l}^{(0.5)}(\zeta, \tau) \quad T_{l, 5}^{*}(\zeta, \tau)=3 \gamma I_{l}^{(2)}(\zeta, \tau) \\
& T_{l, 6}^{*}(\zeta, \tau)=1.2 \gamma\left[I_{l}^{(0)}(\zeta, \tau)+I_{l}^{(1)}(\zeta, \tau)-2 I_{l}^{(2)}(\zeta, \tau)\right] \\
& T_{l, 7}^{*}(\zeta, \tau)=1.2 \gamma\left[3 I_{l}^{(1)}(\zeta, \tau)-2 I_{l}^{(2)}(\zeta, \tau)\right]
\end{aligned}
$$

where $(l=1,2, \alpha=0,0.5,1,2,3)$

$$
I_{l}^{(\alpha)}(\zeta, \tau)=\int_{0}^{\tau} \frac{1}{\sqrt{\pi(\tau-s)}}\left(\frac{s}{\tau_{s}}\right)^{\alpha} \exp \left[-Z_{l}^{2}(\zeta, \tau-s)\right] d s
$$

Using substitution $x=1 / \sqrt{\tau-s}$, we write integrals (3.6) in the form

$$
\begin{aligned}
& I_{l}^{(0)}(\zeta, \tau)=J_{l}^{(2)}(\zeta, \tau) \quad I_{l}^{(0.5)}(\zeta, \tau)=\sqrt{\tau^{*}} M_{l}(\zeta, \tau) \\
& I_{l}^{(1)}(\zeta, \tau)=\tau^{*} J_{l}^{(2)}(\zeta, \tau)-J_{l}^{(4)}(\zeta, \tau) / \tau^{s} \\
& I_{l}^{(2)}(\zeta, \tau)=\tau^{* 2} J_{l}^{(2)}(\zeta, \tau)-2 \tau^{*} J_{l}^{(4)}(\zeta, \tau) \tau_{s}+J_{l}^{(6)}(\zeta, \tau) / \tau_{s}^{2} \\
& I_{l}^{(3)}(\zeta, \tau)=\tau^{*} s J_{l}^{(2)}(\zeta, \tau)-3 \tau^{* 2} J_{l}^{(4)}(\zeta, \tau) / \tau_{s}+3 \tau^{*} J_{l}^{(6)}(\zeta, \tau) / \tau_{s}^{2}-J_{l}^{(8)}(\zeta, \tau) / \tau_{s}^{3}
\end{aligned}
$$

where $(l=1,2)$

$$
\begin{aligned}
& J_{l}^{(n)}(\zeta, \tau)=\frac{2}{\sqrt{\pi}} \int_{1 / \sqrt{\tau}}^{\infty} x^{-n} \exp \left[-\tau Z_{l}^{2}(\zeta, \tau) x\right] d x \quad n=2,4,6,8 \\
& M_{l}(\zeta, \tau)=\frac{1}{\sqrt{\pi}} \int_{1 \sqrt{\tau}}^{\infty} x^{-2} \sqrt{x-\tau^{-1}} \exp \left[-\tau Z_{l}^{2}(\zeta, \tau) x\right] d x
\end{aligned}
$$


Integrals $(3.8)_{1}$ were determined by means of the recursive relation (Prudnikov et al., 1986) $(l=1,2)$

$$
\begin{aligned}
& J_{l}^{(n)}(\zeta, \tau)=\tau\left\{\frac{2}{\sqrt{\pi}} \sqrt{\tau^{n-3}} \exp \left[-Z_{l^{2}}(\zeta, \tau)\right]-\frac{2}{n-1} Z_{l}^{2}(\zeta, \tau) J_{l}^{(n-2)}(\zeta, \tau)\right\} \quad n=4,6,8 \\
& J_{l}^{(2)}(\zeta, \tau)=2 \sqrt{\tau} \operatorname{ierfc} Z_{l}(\zeta, \tau)
\end{aligned}
$$

Substituting the found functions $J_{l}^{(n)}(\zeta, \tau)(3.9)$ to the right sides of formulas (3.7), we obtain

$$
\begin{aligned}
& I_{l}^{(0)}(\zeta, \tau)=2 \sqrt{\tau} \operatorname{ierfc} Z_{l}(\zeta, \tau) \\
& I_{l}^{(1)}(\zeta, \tau)=\frac{2}{3} \sqrt{\tau} \tau^{*}\left\{2\left[1+Z_{l}^{2}(\zeta, \tau)\right] \operatorname{ierfc} Z_{l}(\zeta, \tau)-Z_{l}(\zeta, \tau) \operatorname{erfc} Z_{l}(\zeta, \tau)\right\} \\
& I_{l}^{(2)}(\zeta, \tau)=\frac{2}{15} \sqrt{\tau} \tau^{* 2}\left\{\left[8+18 Z_{l}^{2}(\zeta, \tau)+4 Z_{l}^{4}(\zeta, \tau)\right] \operatorname{ierfc} Z_{l}(\zeta, \tau)\right. \\
& \left.-\left[7+2 Z_{l}^{2}(\zeta, \tau)\right] \operatorname{erfc} Z_{l}(\zeta, \tau)\right\} \\
& I_{l}^{(3)}(\zeta, \tau)=\frac{2}{105} \sqrt{\tau} \tau^{* 3}\left\{\left[48+174 Z_{l}^{2}(\zeta, \tau)+80 Z_{l}^{4}(\zeta, \tau)+8 Z_{l}^{6}(\zeta, \tau)\right] \operatorname{ierfc} Z_{l}(\zeta, \tau)\right. \\
& \left.-(\zeta, \tau)\left[57+36 Z_{l}^{2}(\zeta, \tau)+4 Z_{l}^{4}(\zeta, \tau)\right] \operatorname{erfc} Z_{l}(\zeta, \tau)\right\}
\end{aligned}
$$

where the functions $Z_{l}(\zeta, \tau), l=1,2$ have form (3.2). Integration by parts in formula $(3.8)_{2}$ gives

$$
\begin{array}{ll}
M_{l}(\zeta, \tau)=0.5 M_{l}^{(0)}(\zeta, \tau)-\tau Z_{l}^{2}(\zeta, \tau) M_{l}^{(1)}(\zeta, \tau) \quad l=1,2 & \\
M_{l}^{(n)}(\zeta, \tau)=\frac{1}{\sqrt{\pi}} \int_{1 / \tau}^{\infty} x^{-1}\left(x-\tau^{-1}\right)^{n-1 / 2} \exp \left[-\tau Z_{l}^{2}(\zeta, \tau) x\right] d x & n=0,1
\end{array}
$$

Taking into account that (Prudnikov et al., 1986) $(u>0, \operatorname{Re} \nu>-1, \operatorname{Re} \mu>0)$

$$
\int_{u}^{\infty} x^{-1}(x-u)^{\nu} \exp (-\mu x) d x=u^{\nu} \Gamma(\nu+1) \Gamma(-\nu, u \mu)
$$

where $\Gamma(x)$ - gamma function, $\Gamma(\nu, x)$ - incomplete gamma function, we rewrite integrals $(3.11)_{2}$ in the form

$$
M_{l}^{(n)}(\zeta, \tau)=\tau^{1 / 2-n} \Gamma(0.5+n) \Gamma\left[0.5-n, Z_{l}^{2}(\zeta, \tau)\right] / \sqrt{\pi} \quad l=1,2 \quad n=0,1
$$

Taking into account the relations (Abramowitz and Stegun, 1964)

$$
\begin{aligned}
& \Gamma(0.5)=\sqrt{\pi} \quad \Gamma(1.5)=0.5 \sqrt{\pi} \quad \Gamma\left[0.5, Z_{l}^{2}(\zeta, \tau)\right]=\sqrt{\pi} \operatorname{erfc} Z_{l}(\zeta, \tau) \\
& \Gamma\left[-0.5, Z_{l}^{2}(\zeta, \tau)\right]=2 \sqrt{\pi}\left[Z_{l}(\zeta, \tau)\right]^{-1} \operatorname{ierfc} Z_{l}(\zeta, \tau)
\end{aligned}
$$

from formula (3.13), we obtain $(l=1,2)$

$$
M_{l}^{(0)}(\zeta, \tau)=\sqrt{\pi \tau} \operatorname{erfc} Z_{l}(\zeta, \tau) \quad M_{l}^{(1)}(\zeta, \tau)=\sqrt{\pi}\left[\sqrt{\tau} Z_{l}(\zeta, \tau)\right]^{-1} \operatorname{ierfc} Z_{l}(\zeta, \tau)
$$

Substituting the function $M_{l}^{(n)}(\zeta, \tau), n=0,1$ (3.15) to the right side of equation $(3.11)_{1}$, we first found

$$
M_{l}(\zeta, \tau)=0.5 \sqrt{\pi \tau}\left[\operatorname{erfc} Z_{l}(\zeta, \tau)-2 Z_{l}(\zeta, \tau) \operatorname{ierfc} Z_{l}(\zeta, \tau)\right] \quad l=1,2
$$


and then, after substitution of the functions $M_{l}(\zeta, \tau)(3.16)$ to the second of formulas (3.7), we achieve

$$
I_{l}^{(0.5)}(\zeta, \tau)=0.5 \sqrt{\pi \tau \tau^{*}}\left[\operatorname{erfc} Z_{l}(\zeta, \tau)-2 Z_{l}(\zeta, \tau) \operatorname{ierfc} Z_{l}(\zeta, \tau)\right] \quad l=1,2
$$

Taking into account the functions $I_{l}^{(\alpha)}(\zeta, \tau), l=1,2, \alpha=0,0.5,1,2,3(3.10)$ and (3.17) in formulas (3.5), we determine the dimensionless temperatures $T_{l, i}^{*}(\zeta, \tau), l=1,2, i=1,2, \ldots, 7$ in the final form

$$
\begin{aligned}
& T_{l, 1}^{*}(\zeta, \tau)=\frac{4}{3} \gamma \sqrt{\tau} \tau^{*}\left\langle\left\{3 / \tau-2\left[1+Z_{l}^{2}(\zeta, \tau)\right]\right\} \operatorname{ierfc} Z_{l}(\zeta, \tau)+Z_{l}(\zeta, \tau) \operatorname{erfc} Z_{l}(\zeta, \tau)\right\rangle \\
& T_{l, 2}^{*}(\zeta, \tau)=\frac{4}{3} \gamma \sqrt{\tau} \tau^{*}\left\{2\left[1+Z_{l}^{2}(\zeta, \tau)\right] \operatorname{ierfc} Z_{l}(\zeta, \tau)-Z_{l}(\zeta, \tau) \operatorname{erfc} Z_{l}(\zeta, \tau)\right\} \\
& T_{l, 3}^{*}(\zeta, \tau)=\gamma\left\langle 3 \sqrt{\tau} \operatorname{ierfc} Z_{l}(\zeta, \tau)-0.5 \sqrt{\tau} \tau^{*}\left\{2\left[1+Z_{l}^{2}(\zeta, \tau)\right] \operatorname{ierfc} Z_{l}(\zeta, \tau)\right.\right. \\
& \left.\quad-Z_{l}(\zeta, \tau) \operatorname{erfc} Z_{l}(\zeta, \tau)\right\}-\frac{\sqrt{\tau} \tau^{*} r}{40}\left\{\left[8+18 Z_{l^{2}}(\zeta, \tau)+4 Z_{l}^{4}(\zeta, \tau)\right] \operatorname{ierfc} Z_{l}(\zeta, \tau)\right. \\
& \left.\quad-Z_{l}(\zeta, \tau)\left[7+2 Z_{l}^{2}(\zeta, \tau)\right] \operatorname{erfc} Z_{l}(\zeta, \tau)\right\}-\frac{\sqrt{\tau} \tau^{* 3}}{560}\left\{48+174 Z_{l}^{2}(\zeta, \tau)+80 Z_{l}^{4}(\zeta, \tau)\right. \\
& \left.\left.\quad+8 Z_{l}^{6}(\zeta, \tau) \operatorname{ierfc} Z_{l}(\zeta, \tau)-Z_{l}(\zeta, \tau)\left[57+36 Z_{l}^{2}(\zeta, \tau)+4 Z_{l}^{4}(\zeta, \tau)\right] \operatorname{erfc} Z_{l}(\zeta, \tau)\right\}\right\rangle \\
& T_{l, 4}^{*}(\zeta, \tau)=\frac{3}{4} \gamma \sqrt{\pi \tau \tau^{*}}\left[\operatorname{erfc} Z_{l}(\zeta, \tau)-2 Z_{l}(\zeta, \tau) \operatorname{ierfc} Z_{l}(\zeta, \tau)\right] \\
& T_{l, 5}^{*}(\zeta, \tau)=\frac{2}{5} \gamma \sqrt{\tau} \tau^{* 2}\left\{\left[8+18 Z_{l}^{2}(\zeta, \tau)+4 Z_{l}^{4}(\zeta, \tau)\right] \operatorname{ierfc} Z_{l}(\zeta, \tau)\right. \\
& \left.\quad-Z_{l}(\zeta, \tau)\left[7+2 Z_{l}^{2}(\zeta, \tau)\right] \operatorname{erfc} Z_{l}(\zeta, \tau)\right\} \\
& T_{l, 6}^{*}(\zeta, \tau)=\frac{4}{5} \gamma \sqrt{\tau}\left\langle 3 \operatorname{ierfc} Z_{l}(\zeta, \tau)+\tau^{*}\left\{2\left[1+Z_{l}^{2}(\zeta, \tau)\right] \operatorname{ierfc} Z_{l}(\zeta, \tau)\right.\right. \\
& \left.\quad-Z_{l}(\zeta, \tau) \operatorname{erfc} Z_{l}(\zeta, \tau)\right\}-0.4 \tau^{* 2}\left\{\left[8+18 Z_{l}^{2}(\zeta, \tau)+4 Z_{l}^{4}(\zeta, \tau)\right] \operatorname{ierfc} Z_{l}(\zeta, \tau)\right. \\
& \left.\left.\quad-Z_{l}(\zeta, \tau)\left[7+2 Z_{l}^{2}(\zeta, \tau)\right] \operatorname{erfc} Z_{l}(\zeta, \tau)\right\}\right\rangle \\
& T_{l, 7}^{*}(\zeta, \tau)=\frac{4}{5} \gamma \sqrt{\tau} \tau^{*}\left\langle 3\left\{2\left[1+Z_{l}^{2}(\zeta, \tau)\right] \operatorname{ierfc} Z_{l}(\zeta, \tau)-Z_{l}(\zeta, \tau) \operatorname{erfc} Z_{l}(\zeta, \tau)\right\}\right. \\
& \quad-0.4 \tau^{*}\left\{\left[8+18 Z_{l}^{2}(\zeta, \tau)+4 Z_{l}^{4}(\zeta, \tau)\right] \operatorname{ierfc} Z_{l}(\zeta, \tau)\right. \\
& \left.\left.\quad-Z_{l}(\zeta, \tau)\left[7+2 Z_{l}^{2}(\zeta, \tau)\right] \operatorname{erfc} Z_{l}(\zeta, \tau)\right\}\right\rangle
\end{aligned}
$$

On the contact surface $\zeta=0$, from formulas (3.18) it follows that $T_{1, i}^{*}(0, \tau)=T_{2, i}^{*}(0, \tau) \equiv$ $T_{i}^{*}(\tau), 0 \leqslant \tau \leqslant \tau_{s}, i=1,2, \ldots, 7$, where

$$
\begin{aligned}
& T_{1}^{*}(\tau)=4 \gamma \sqrt{\frac{\tau}{\pi}}\left(1-\frac{2}{3} \tau^{*}\right) \quad T_{2}^{*}(\tau)=\frac{8}{3} \gamma \sqrt{\frac{\tau}{\pi}} \tau^{*} \\
& T_{3}^{*}(\tau)=\gamma \sqrt{\frac{\tau}{\pi}}\left(3-\tau^{*}-\frac{1}{5} \tau^{* 2}-\frac{3}{35} \tau^{* 3}\right) \quad T_{4}^{*}(\tau)=\frac{3}{4} \gamma \sqrt{\pi \tau \tau^{*}} \\
& T_{5}^{*}(\tau)=\frac{16}{5} \gamma \sqrt{\tau} \tau^{* 2} \quad T_{6}^{*}(\tau)=\frac{4}{5} \gamma \sqrt{\frac{\tau}{\pi}}\left(3+2 \tau^{*}-\frac{16}{5} \tau^{* 2}\right) \\
& T_{7}^{*}(\tau)=\frac{8}{5} \gamma \sqrt{\frac{\tau}{\pi}} \tau^{*}\left(3-\frac{8}{5} \tau^{*}\right)
\end{aligned}
$$

This suggests that boundary condition (2.7) is satisfied.

According to Fourier's law (Carslaw and Jaeger, 1959), the intensities of heat fluxes in the friction pair elements are equal

$$
q_{l, i}(z, t)=(-1)^{l} K_{l} \frac{\partial T_{l, i}(z, t)}{\partial z} \quad l=1,2 \quad i=1,2, \ldots, 7
$$


Taking expressions (2.2) and (2.6) into account, we write formulae (3.20) in the dimensionless form

$$
q_{l, i}^{*}(\zeta, \tau)=\frac{q_{l, i}(z, t)}{q_{0}}= \begin{cases}-\frac{\partial T_{1, i}^{*}(\zeta, \tau)}{\partial \zeta} & \text { for } \quad \zeta \geqslant 0,0 \leqslant \tau \leqslant \tau_{s} \\ K^{*} \frac{\partial T_{2, i}^{*}(\zeta, \tau)}{\partial \zeta} & \text { for } \quad \zeta \leqslant 0,0 \leqslant \tau \leqslant \tau_{s}\end{cases}
$$

Substituting dimensionless temperatures (3.18) to formulas (3.21), we obtain

$$
\begin{aligned}
& q_{l, 1}^{*}(\zeta, \tau)=2 \eta_{l}\left[2 \tau^{*} Z_{l}(\zeta, \tau) \operatorname{ierfc} Z_{l}(\zeta, \tau)+\left(1-\tau^{*}\right) \operatorname{erfc} Z_{l}(\zeta, \tau)\right] \\
& q_{l, 2}^{*}(\zeta, \tau)=2 \eta_{l} \tau^{*}\left[\operatorname{erfc} Z_{l}(\zeta, \tau)-2 Z_{l}(\zeta, \tau) \operatorname{ierfc} Z_{l}(\zeta, \tau)\right] \\
& q_{l, 3}^{*}(\zeta, \tau)=\eta_{l}\left\langle\tau^{* 2}\left\{\frac{1}{8}\left[5+2 Z_{l}^{2}(\zeta, \tau)\right]+\frac{\tau^{*}}{280}\left[117+98 Z_{l}^{2}(\zeta, \tau)+14 Z_{l}^{4}(\zeta, \tau)\right]\right\}\right. \\
& \cdot Z_{l}(\zeta, \tau) \operatorname{ierfc} Z_{l}(\zeta, \tau)+\frac{3}{2}\left\{1-\tau^{*}\left[0.5-Z_{l}(\zeta, \tau)\right]-\frac{\tau^{* 2}}{24}\left[3+2 Z_{l}^{2}(\zeta, \tau)\right]\right. \\
& \left.\left.\quad-\frac{\tau^{* 3}}{1680}\left[105+168 Z_{l}^{2}(\zeta, \tau)+28 Z_{l}^{4}(\zeta, \tau)\right]\right\} \operatorname{erfc} Z_{l}(\zeta, \tau)\right\rangle \\
& q_{l, 4}^{*}(\zeta, \tau)=1.5 \eta_{l} \sqrt{\pi \tau^{*}} \operatorname{ierfc} Z_{l}(\zeta, \tau) \\
& q_{l, 5}^{*}(\zeta, \tau)=\eta_{l} \tau^{* 2}\left\{\left[3+2 Z_{l}^{2}(\zeta, \tau)\right]\right\} \operatorname{erfc} Z_{l}(\zeta, \tau) \\
& \left.\quad-2\left[5+2 Z_{l}^{2}(\zeta, \tau)\right] Z_{l}(\zeta, \tau) \operatorname{ierfc} Z_{l}(\zeta, \tau)\right\} \\
& q_{l, 6}^{*}(\zeta, \tau)=0.4 \eta_{l}\left\langle\left\{3\left(1+\tau^{*}\right)-2 \tau^{* 2}\left[3+2 Z_{l}^{2}(\zeta, \tau)\right]\right\} \operatorname{erfc} Z_{l}(\zeta, \tau)\right. \\
& \left.\quad-2 \tau^{*}\left\{3-2 \tau^{*}\left[5+2 Z_{l}^{2}(\zeta, \tau)\right] Z_{l}(\zeta, \tau) \operatorname{ierfc} Z_{l}(\zeta, \tau)\right\}\right\rangle \\
& q_{l, 7}^{*}(\zeta, \tau)=0.4 \eta_{l} \tau^{*}\left\langle\left\{9-2 \tau^{*}\left[3+2 Z_{l}^{2}(\zeta, \tau)\right]\right\} \operatorname{erfc} Z_{l}(\zeta, \tau)\right. \\
& \left.\quad-2\left\{9-2 \tau^{*}\left[5+2 Z_{l}^{2}(\zeta, \tau)\right]\right\} Z_{l}(\zeta, \tau) \operatorname{ierfc} Z_{l}(\zeta, \tau)\right\rangle
\end{aligned}
$$

where $\eta_{1}=\gamma, \eta_{2}=\varepsilon \gamma$ and the coefficients $\varepsilon$ and $\gamma$ are calculated from formulas (3.2).

On the contact surface $\zeta=0$, from formulas $(3.22)$ it follows that $q_{l, i}^{*}(0, \tau)=\eta_{l} q_{i}^{*}\left(\tau^{*}\right)$, $0 \leqslant \tau \leqslant \tau_{s}, l=1,2$, where the dimensionless specific powers of friction $q_{i}^{*}\left(\tau^{*}\right), i=1,2, \ldots, 7$ have form (2.3). Hence, we find $q_{1, i}(0, \tau)+q_{2, i}(0, \tau)=q_{i}(\tau), 0 \leqslant \tau \leqslant \tau_{s}, i=1,2, \ldots, 7$, that confirms satisfaction of boundary condition (2.7) by solutions (3.18). Moreover, we conclude that the coefficients $\eta_{l}, l=1,2$ describe the partition of heat fluxes between the elements of the tribocouple in the form proposed by Charron (1943)

$$
\eta_{1} \equiv \frac{q_{1, i}^{*}(0, \tau)}{q_{i}^{*}(\tau)}=\frac{1}{1+\varepsilon} \quad \eta_{2}=1-\eta_{1} \equiv \frac{q_{2, i}^{*}(0, \tau)}{q_{i}^{*}(\tau)}=\frac{\varepsilon}{1+\varepsilon} \quad i=1,2, \ldots, 7
$$

\section{Approximate Chichinadze's solution}

Another variant of the solution to thermal problem of friction (2.7) and (2.8) was proposed by Chichinadze (1967). The method for obtaining that solution with simplifying assumptions was presented by Yevtushenko et al. (2017), too. Based on that solution, with taking expressions (2.6) into account, the dimensionless temperatures in the elements of the friction couple can be written in the form $\left(0 \leqslant \tau \leqslant \tau_{s}\right)$ 


$$
\begin{aligned}
& T_{1, i}^{*}(\zeta, \tau)=\eta_{1}\left\{\left[\frac{a_{1}^{*}}{3}-\zeta\left(1-\frac{\zeta}{2 a_{1}}\right)\right] q_{i}^{*}(\tau)+\frac{\tau_{s}^{*}}{a_{1}} w_{i}^{*}(\tau)\right. \\
& \left.-2 a_{1}^{*} q_{i}^{*}(0) \sum_{n=1}^{\infty} \frac{\exp \left[-\left(n \pi / a_{1}\right)^{2} \tau\right]}{(\pi n)^{2}} \cos \left(\frac{n \pi}{a_{1}^{*}} \zeta\right)\right\} \quad 0 \leqslant \zeta \leqslant a_{1}^{*} \\
& T_{2, i}^{*}(\zeta, \tau)=\frac{\eta_{2}}{K^{*}}\left\{\left[\frac{a_{2}^{*}}{3}+\zeta\left(1+\frac{\zeta}{2 a_{2}^{*}}\right)\right] q_{i}^{*}(\tau)+\frac{k^{*} \tau_{s}}{a_{2}^{*}} w_{i}^{*}(\tau)\right. \\
& \left.-2 a_{2}^{*} q_{i}^{*}(0) \sum_{n=1}^{\infty} \frac{\exp \left[-\left(n \pi / a_{2}^{*}\right)^{2} k^{*} \tau\right]}{(\pi n)^{2}} \cos \left(\frac{n \pi}{a_{2}^{*}} \zeta\right)\right\} \quad-a_{2}^{*} \leqslant \zeta \leqslant 0
\end{aligned}
$$

where

$$
w_{i}^{*}(\tau)=\int_{0}^{\tau} q_{i}^{*}(s) d s \quad i=1,2, \ldots, 7
$$

and $a_{l}^{*}=a_{l} / a, l=1,2$ are heat partition ratios (3.23). It should be noted that solution (4.1) is approximate due to two simplifying assumptions:

- change of temperature in time at any point on the $\zeta$ axis is proportional to the specific power of friction;

- mean temperature of the contact surface of the pad and the disc is the same during the whole braking process.

Substituting functions $q_{i}^{*}(\tau), i=1,2, \ldots, 7$ (2.3) to the right side of the first formula in (4.2) we find the dimensionless density of the friction work in the form

$$
\begin{array}{lrrr}
w_{1}^{*}(\tau) & =\tau^{*}\left(2-\tau^{*}\right) \quad w_{2}^{*}(\tau)=\tau^{*} & w_{3}^{*}(\tau)=1-\sqrt{\left(1-\tau^{*}\right)^{3}} \\
w_{4}(\tau)=\tau^{*} \sqrt{\tau^{*}} & w_{5}^{*}(\tau)=\tau^{* 3} & w_{6}^{*}(\tau)=0.2 \tau^{*}\left(6+3 \tau^{*}-4 \tau^{* 2}\right) \\
w_{7}^{*}(\tau)=0.2 \tau^{* 2}\left(9-4 \tau^{*}\right) &
\end{array}
$$

On the contact surface $\zeta=0$, solutions (4.1) lead to (Chichinadze, 2009) $\left(0 \leqslant \tau \leqslant \tau_{s}\right)$

$$
\begin{aligned}
& T_{1, i}^{*}(0, \tau)=\eta_{1}\left[\frac{a_{1}^{*}}{3} q_{i}^{*}(\tau)+\frac{\tau_{s}}{a_{1}^{*}} w_{i}^{*}(\tau)-2 a_{1}^{*} q_{i}^{*}(0) \sum_{n=1}^{\infty} \exp \left[-\left(\frac{n \pi}{a_{1}^{*}}\right)^{2} \tau\right] \frac{1}{(\pi n)^{2}}\right] \\
& T_{2, i}^{*}(0, \tau)=\frac{\eta_{2}}{K^{*}}\left[\frac{a_{2}^{*}}{3} q_{i}^{*}(\tau)+\frac{k^{*} \tau_{s}}{a_{2}^{*}} w_{i}^{*}(\tau)-2 a_{2}^{*} q_{i}^{*}(0) \sum_{n=1}^{\infty} \exp \left[-\left(\frac{n \pi}{a_{1}^{*}}\right)^{2} \tau\right] \frac{1}{(\pi n)^{2}}\right]
\end{aligned}
$$

where functions $q_{i}^{*}(\tau)$ and $w_{i}^{*}(\tau), i=1,2, \ldots, 7$ are determined from formulas (2.3) and (4.3), respectively.

\section{Results of calculations}

Numerical analysis has been performed for the braking system in which the disc was made of alloyed cast iron ChNMKh $\left(K_{1}=51 \mathrm{~W} /(\mathrm{mK}), k_{1}=14 \cdot 10^{-6} \mathrm{~m}^{2} / \mathrm{s}\right)$ and material of the pad was retinax FM-16L $\left(K_{2}=0.65 \mathrm{~W} /(\mathrm{mK}), k_{2}=0.4 \cdot 10^{-6} \mathrm{~m}^{2} / \mathrm{s}\right)$ (Chichinadze et al., 1986). For those materials, we calculated the values of dimensionless thermal conductivity $K^{*}=0.0127$ and thermal diffusivity $k^{*}=0.0286$, and next, the coefficient of thermal activity $\varepsilon=0.0754$ as well as heat partition ratios $\eta_{1}=0.93, \eta_{2}=0.07$. The linear parameter $a$, used in application of the dimensionless variables in formulas (2.6) is assumed as the effective depth $a_{1}$ of heat 
penetration in the disc. Then $a_{1}^{*}=1, a_{2}^{*}=a_{2} / a_{1}=\sqrt{k^{*}}=0.169$, and the dimensionless braking time is $\tau_{s}=0.33$. The dimensionless input parameters used in the calculations are the spatial coordinate $\zeta$ and the time $\tau$, Eqs. (2.6).

The changes over the braking time $0 \leqslant \tau \leqslant \tau_{s}$, dimensionless temperatures $T_{i}^{*}(\tau)$, $i=1,2, \ldots, 7$ (3.19) and the intensities of heat fluxes $q_{l, i}^{*}(0, \tau)=\eta_{l} q_{i}^{*}(\tau), l=1,2$ with dimensionless specific powers of friction $q_{i}^{*}(\tau), i=1,2, \ldots, 7,(2.3)$, are shown by solid lines in Fig. 1. The corresponding results for temperatures obtained from approximate solutions (4.4) are presented by dashed lines. Let us note that for the selected friction pair, $93 \%$ of the generated heat enters the disc $(l=1)$, and the rest $7 \%$ - the pad $(l=2)$. Therefore, the evolutions of heat fluxes intensities $q_{1, i}^{*}(0, \tau)$ are almost convergent with the temporal profiles of the specific power of friction $q_{i}^{*}(\tau), i=1,2, \ldots, 7$, Eqs. (2.3). These profiles can be classified into one of three groups.
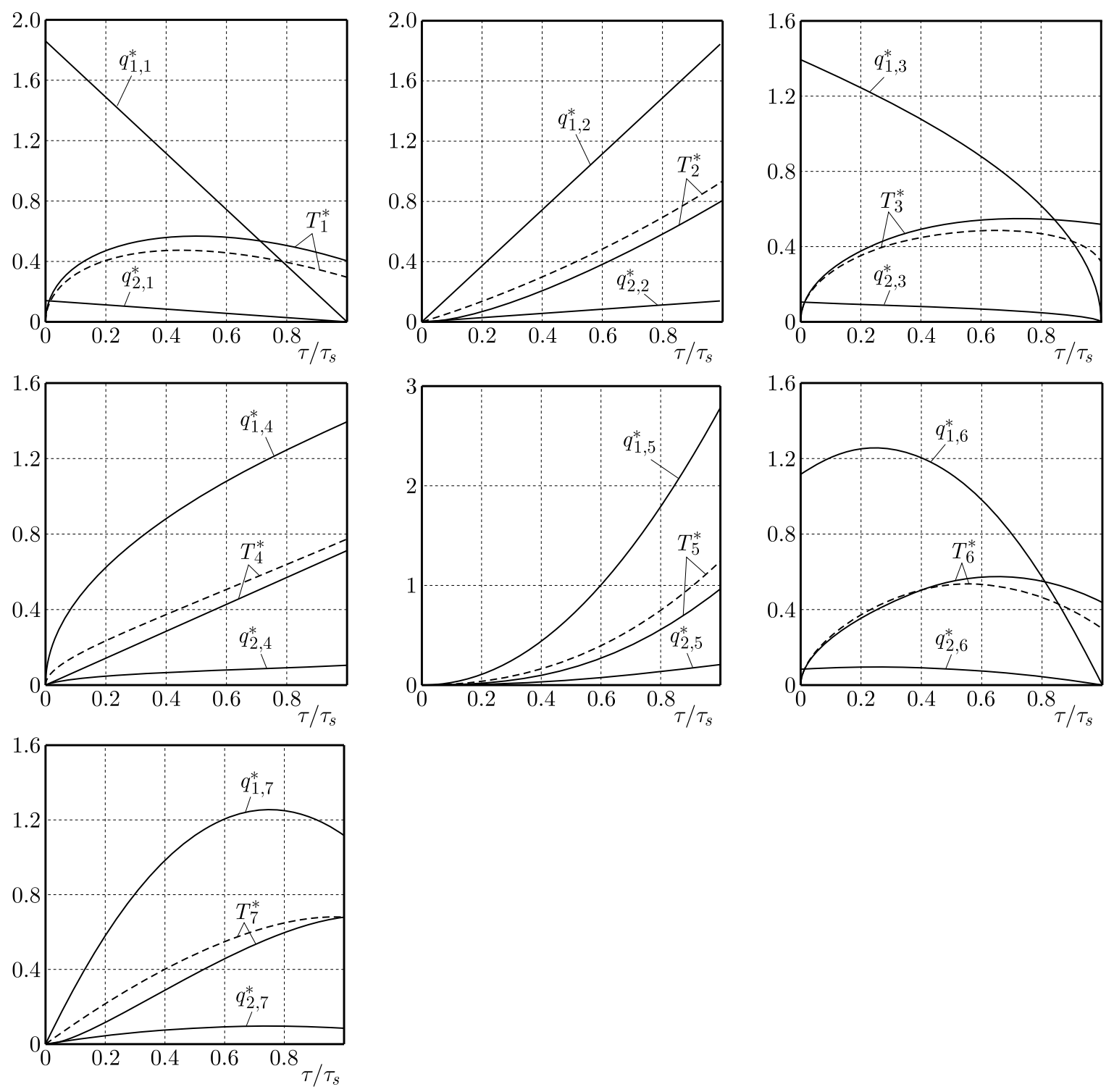

Fig. 1. Evolutions of the dimensionless temperatures $T_{i}^{*}$ and the intensities of heat fluxes $q_{l, i}^{*}, l=1,2$, $i=1,2, \ldots, 7$ on the contact surface $\zeta=0$. Solid lines - results obtained by means of exact solutions (3.19), dashed lines - based on approximate solution (4.4) 
The first group contains graphs of the intensities of heat fluxes $q_{i}^{*}(0, \tau), i=1,3$, which achieve the maximum values at the initial time moment $\tau=0$ and then monotonically decrease to zero at the stopping moment $\tau=\tau_{s}$. Belonging to this group, the evolutions of dimensionless temperatures increase in the initial stage of the braking process, afterwards achieve maximum values and, subsequently, undergo slight cooling of the contact surface. The specific power of friction $q_{1}^{*}(\tau)$ linearly reduces with time and the corresponding dimensionless temperature reaches its maximum $T_{1, \max }^{*}=0.57$ in the middle of the braking process time $\tau=0.5 \tau_{s}$. Such variation of the friction power and temperature is characteristic for braking with a constant deceleration (Chichinadze, 1967). The graph of the function $q_{3}^{*}(\tau)$ is a decreasing concave parabola, and the maximum temperature $T_{3, \max }^{*}=0.55$ is achieved at $\tau=0.74 \tau_{s}$. The evolutions of dimensionless temperatures for this group of specific friction power, found from approximate Chichinadze's solution, are qualitatively close to each other. In quantitative terms, the approximate solution gives understated values of temperatures (up to $39 \%$ for $i=3$ at the stop) in comparison with the values obtained on the basis of the exact solutions.

The second group includes the specific power of friction $q_{i}^{*}(\tau), i=2,4,5$, which monotonically increases from zero at the initial time moment to the maximum value at the stopping moment. Specific for the evolutions of temperature in this group is that they are also monotonically increasing functions which reach the maximum values $T_{i, \max }^{*}=0.81,0.71,0.97, i=2,4,5$ at the stopping moment. As in the first group, the course of changes of dimensionless temperatures over time found by means of the approximate solution is similar to the time profile of temperatures determined on the basis of the accurate solutions. However, in quantitative terms, the approximate results overestimates the values of temperature (up to $28 \%$ for $i=5$ at $\tau=\tau_{s}$ ) with respect to data from the exact solutions.

The third group comprises temporal profiles of the dimensionless specific heat power $q_{i}^{*}(\tau)$, $i=6,7$, which have a local maximum within the braking time interval $0 \leqslant \tau \leqslant \tau_{s}$. The dimensionless temperature $T_{6}^{*}(\tau)$ rises at the beginning of the braking process, then reaches the $\operatorname{maximum} T_{i, \max }^{*}=0.58$ at the moment $\tau=0.66 \tau_{s}$ and next, insignificant cooling of the contact surface follows until the stopping moment. Temperature $T_{7}^{*}(\tau)$ in the braking process increases monotonically to the maximum value $T_{7, \max }^{*}=0.68$, achieved at the moment of standstill. Differences between the results obtained on the basis of the exact and approximate Chichinadze's solutions are the most noticeable in this group. At the stage of heating (cooling) of the contact surface, the temperature $T_{6}^{*}(\tau)$ achieved from the approximate solution are higher (lower) than the corresponding values found by means of the exact solution. At the same time, for $i=7$, the temperature from the inaccurate solution is overstated with respect to the temperature calculated by means of the accurate solution.

Isolines of the dimensionless temperatures $T_{l, i}^{*}(\zeta, \tau), l=1,2, i=1,2, \ldots, 7$ calculated by means of exact (3.18) (solid lines) and approximate (4.1) (dashed lines) solutions, are presented in Fig. 2. We see that the influence of the specific friction power on the distributions of temperature fields is much larger in the disc than in the pad. This is caused by the fact that retinax is a significantly worse thermal conductor in comparison with cast iron. Hence, the effective depth of heat penetration of the pad is smaller $\left(a_{2}^{*}=0.169\right)$ than the corresponding depth of the disc $\left(a_{1}^{*}=1\right)$, and the temperature isolines are more concentrated in the pad than in the disc. However, the spatiotemporal temperature distributions in the disc are determined by the adequate temporal profiles of the specific power of friction. The highest concentration of the isotherms occurs inside the disc adjacent to the heated surface, near the time moments of the maximum achievement. In the first case, from the above mentioned groups of the profiles $q_{i}^{*}(\tau)$, $i=1,3$ at the initial stage of braking, heat penetrates the disc to the effective depth. At the same time, with the increasing distance from the contact surface, the temperature decreases nonlinearly. The distribution of the isotherms inside the disc is different when the heat on the contact surface is generated according to the specific power of friction $q_{i}^{*}(\tau), i=2,4,5$. With 
the increasing depth, the temperature decrease is almost linear and the disc is not heated on the whole effective depth. In the case of the third group of temporal profiles of the specific heat power $q_{i}^{*}(\tau), i=6,7$, the heat penetrates to the effective depth only for $i=6$. Differences between the temperatures obtained by means of exact (3.18) and approximate solutions (4.1) rise along with the increase of the distance from the contact surface. However, in contrast to the evolutions of these temperatures on the friction surface (Fig. 1), their changes over time inside the disc differ significantly, not only quantitatively but also qualitatively (Fig. 2).
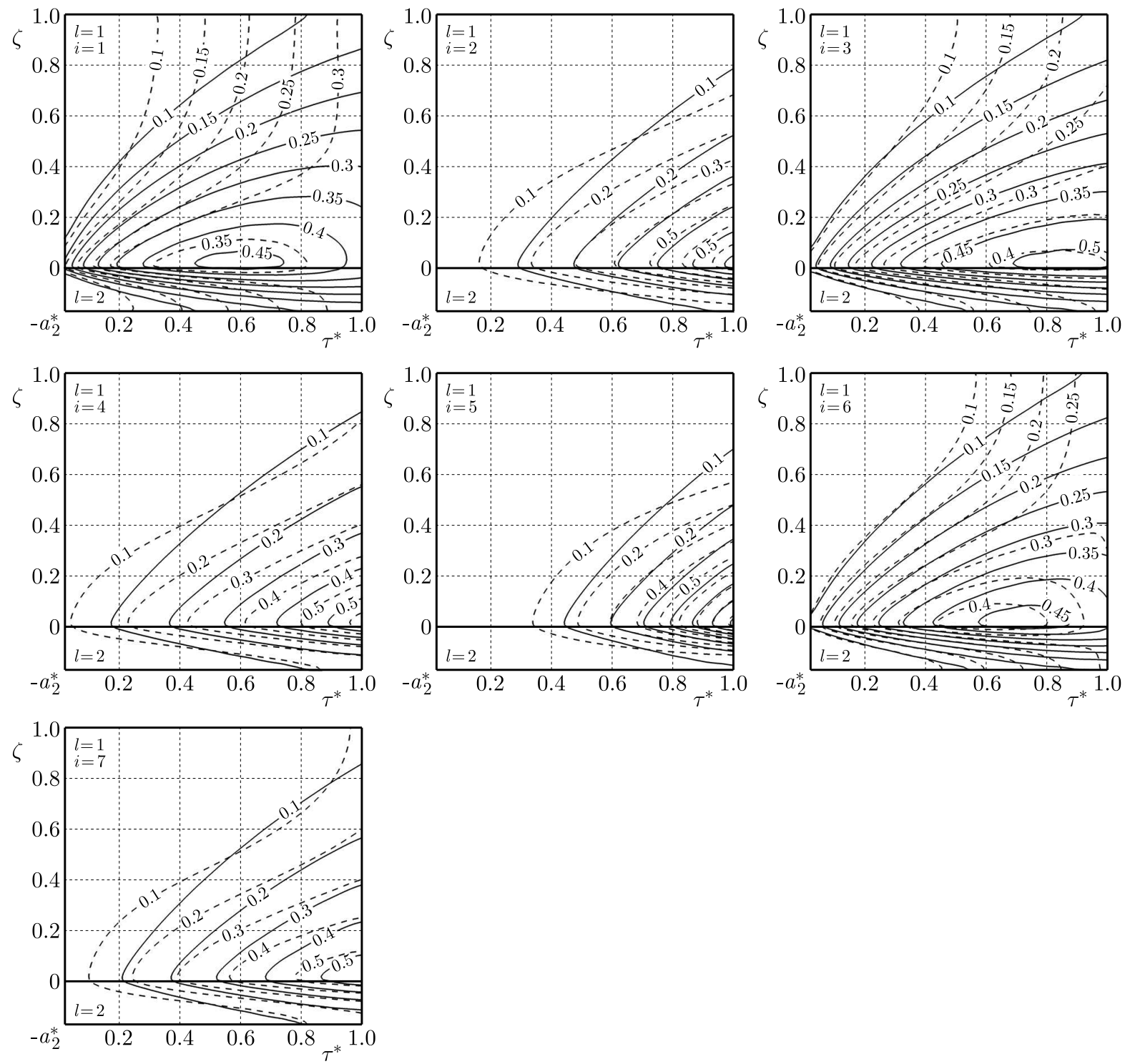

Fig. 2. Isolines of the dimensionless temperatures $T_{l, i}^{*}(\zeta, \tau), i=1,2, \ldots, 7$ in the disc $(l=1)$ and the pad

$(l=2)$. Solid lines - results obtained by means of exact solutions $(3.18)$, dashed lines - based on approximate solution (4.1)

\section{Conclusions}

The influence of the specific friction power on temperature in the disc and the pad during single braking has been investigated. For this aim, seven different time profiles of the specific heat power were selected (Chichinadze, 1967), which were established experimentally. Processes with an equal work of friction in the same braking time were considered. As the standard model, 
a one-dimensional boundary-value problem of heat conduction for two sliding semi-spaces was formulated with taking into account heat generation according to selected temporal profiles of the specific power of friction. The exact solutions to this problem were obtained using Duhamel's theorem. Analytical formulas were obtained, which allowed one to determine spatial-temporal distributions of temperature and heat flux intensities in friction elements. Numerical analysis was carried out for the cast iron disc and the pad made of retinax. Temperature values obtained for that friction pair by means of accurate solutions were compared with corresponding temperatures found by using the approximate solution (Chichinadze, 1967, 2009).

Based on numerical analysis, it was established that the time profile of the specific power of friction had an essential effect on the evolution of temperature on the disc-pad contact area, and also on the spatial-temporal distributions of temperature inside the elements of the friction couple. There was a direct relation between the maximum values of the specific power of friction and the temperature - the longer the time of reaching the maximum values of temperature, the longer time of occurrence of the maximum intensity of the corresponding heat flux. It was shown that inaccurate Chichinadze's solution causes approximately $13 \%$ error in estimating the maximum temperature on the contact surface of the pad and the disc, and it can be used to calculate the average temperature of that surface in braking systems. However, making use of this solution to determine temperature distributions inside the friction elements can cause significant errors during predicting their volumetric temperature.

In addition, it is necessary to note that the actuality of these type of solutions has been proved by the fact that they are used as an "initial approximation" in the development of iterative algorithms for solving relevant non-linear thermal problems of friction for elements made of thermosensitive materials (Yevtushenko et al., 2015).

\section{Acknowledgement}

This article was supported by the National Science Centre of Poland (research project No. 2017/27/B/ST8/01249).

\section{References}

1. Abramowitz M., Stegun I.A., 1964, Handbook of mathematical functions with formulas, graphs and mathematical tables. National Bureau of Standards, Applied Mathematics, Series 55

2. Barber J.R., Comninou M., 1989, Thermoelastic contact problems, [In:] Thermal Stresses III, R. Hetnarski (Edit.), North Holland, Amsterdam, 1-106

3. Blok H., 1955, The dissipation of friction heat, Applied Scientific Research, A, 2-3, 151-181

4. Carslaw H.S., Jaeger J.C., 1959, Conduction of Heat in Solids, 2nd ed., Clarendon Press, Oxford

5. Charron F., 1943, Partage de la chaleur entre deux corps frottants, Publications Scientifiques et Techniques du Ministere de L'air, article 182

6. Chichinadze A.V., 1967, Calculation and Study of External Friction During Braking (in Russian), Nauka, Moscow

7. Chichinadze A.V., 2009, Theoretical and practical problems of thermal dynamics and simulation of the friction and wear of tribocouples, Journal of Friction and Wear, 3, 275-295

8. Chichinadze A.V., Braun E.D., Ginzburg A.G., Ignateva E.V., 1979, Calculation, Testing, and Selection of Frictional Pairs (in Russian), Nauka, Moscow

9. Chichinadze A.V., Matveevski R.M., Braun E.P., 1986, Materials in Tribotechnics Non-Stationary Processes (in Russian), Nauka, Moscow 
10. Fazekas G.A.G., 1953, Temperature gradients and heat stresses in brake drums, SAE Transactions, 61, 279-284

11. Ling F.F., 1959, A quasi-iterative method for computing interface temperature distributions, Zeitschrift für angewandte Mathematik und Physik ZAMP, 10, 461-474

12. Luikov A.V., 1968, Analytical Heat Diffusion Theory, Academic Press, New York

13. Matysiak S.J., Yevtushenko A.A., Ivanyk E.G., 2002, Contact temperature and wear of composite friction elements during braking, International Journal of Heat and Mass Transfer, 45, 193-199

14. Newcomb T.P., Spurr R.T., 1967, Braking of Road Vehicles, Chapman and Hall, London

15. Nosko A.L., Nosko A.P., 2006, Solution of contact heating problem with account for heat transfer between the friction members, Journal of Friction and Wear, 30, 615-625

16. Prudnikov A.P., Brychkov Yu.A., Marichev O.I., 1986, Integrals and series. Elementary functions, Gordon and Breach, New York

17. Sazonov V.S., 2006, Exact solution of the problem of nonstationary heat conduction for two semi-spaces in nonideal contact, Journal of Engineering Physics and Thermophysics, 79, 928-930

18. SAzONOV V.S., 2008, Nonideal contact problem of nonstationary heat conduction for two half-spaces, Journal of Engineering Physics and Thermophysics, 81, 397-408

19. Topczewska K., 2017, Influence of friction power on temperature in the process of braking, Materials Science, 53, 2, 235-242

20. Yevtushenko A.A., Ivanyk E.G., Yevtushenko O.O., 1999, Exact formulae for determination of the mean temperature and wear during braking, Heat and Mass Transfer, 35, 163-169

21. Yevtushenko A.A., Kuciej M., 2012, One-dimensional thermal problem of friction during braking: the history of development and actual state, International Journal of Heat and Mass Transfer, 55, 4118-4153

22. Yevtushenko A.A., Kuciej M., Yevtushenko O., 2013, The boundary conditions on the sliding surface in one-dimensional transient heat problem of friction, International Journal of Heat and Mass Transfer, 59, 1-8

23. Yevtushenko A., Kuciej M., Och E., 2014a, Influence of thermal sensitivity of the pad and disk materials on the temperature during braking, International Communications in Heat and Mass Transfer, 55, 84-92

24. Yevtushenko A., Kuciej M., Och E., 2014b, Temperature in thermally nonlinear pad-disk brake system, International Communications in Heat and Mass Transfer, 57, 274-281

25. Yevtushenko A., Kuciej M., Och E., 2015, Some methods for calculating temperature during the friction of thermosensitive materials, Numerical Heat Transfer. Part A, 67, 696-718

26. Yevtushenko A., Kuciej M., Och E., 2016, Effect of the thermal sensitivity in modeling of the frictional heating during braking, Advances in Mechanical Engineering, 8, 12, 1-10

27. Yevtushenko A., Kuciej M., Topczewska K., 2017, Analytical model for investigation of the effect of friction power on temperature in the disk brake, Advances in Mechanical Engineering, 9, $12,1-12$ 